[0212-7199 (2008) 25: 1; pp 41-43] ANALES DE MEDICINA INTERNA Copyright (C) 2008 ARAN EDICIONES, S.L.

AN. MED. INTERNA (Madrid) Vol. 25, N. $^{\circ} 1$, pp. $41-43,2008$

\title{
¿Definimos correctamente la EPOC?
}

\author{
R. DE LA FUENTE CID, F. J. GONZÁLEZ BARCALA', A. POSE REINO, \\ L. VALDÉS CUDRADO ${ }^{1}$ \\ Servicio de Medicina Interna. ${ }^{\prime}$ Servicio de Neumología. Complejo Hospitalario de \\ Santiago de Compostela. A Coruña
}

DO WE DEFINE THE COPD CORRECTLY?

\begin{abstract}
RESUMEN
La Enfermedad Pulmonar Obstructiva Crónica (EPOC) es una enfermedad muy frecuente, cuya prevalencia sigue aumentando y que en un futuro próximo supondrá un problema socio-sanitario. El propósito de este artículo es hacer una reflexión sobre el concepto EPOC, para contribuir por parte de los médicos a su divulgación a la población y muy concretamente al abandono del hábito tabáquico. Por otro lado también queremos hacer constar, que debido a los avances en el conocimiento etiológico, fisiopatológico, técnicas de imagen etc, el término se ha perfilado de forma muy positiva. No obstante creemos que el acrónimo todavía incluye entidades con perfiles muy diferentes. Esperemos que en el futuro, estableciendo distintos fenotipos y sobre todo con ayuda de la biología molecular podamos clarificar más este concepto.
\end{abstract}

PALABRAS CLAVE: EPOC. Definición. Espirometría.

\begin{abstract}
Chronic obstructive pulmonary disease (COPD) is a very common disease which prevalence is increasing and in a nearly future it will represent a socio-sanitary problem. This article's objective is to make a reflexion about the concept COPD, for the physicians contribute to their divulgation to the population and very specially to help to the tobacco desertion. Besides we want to reveal that the term has been well-finished due to the advance in the knowledge of aetiology, physiopathology, and radiology techniques. Although, we think that the acronym still includes entities with a very different features. We hope to clarify this concept in the future, establishing different phenotypes and mainly with molecular biology.
\end{abstract}

KEY WORDS: COPD. Definition. Spirometry.

De la Fuente Cid R, González Barcala FJ, Pose Reino A, Valdés Cuadrado L. ¿Definimos correctamente la EPOC? An Med Interna (Madrid) 2008; 25: 41-43.

\section{INTRODUCCIÓN}

El primer intento de definir y darle categoría a la EPOC es en 1958 en el Simposio Ciba en el que se introduce el concepto de enfermedad pulmonar crónica no específica, en el cual se incluye la bronquitis crónica y la enfermedad obstructiva generalizada, que a su vez engloba el asma y el enfisema (1). En 1965 la bronquitis crónica se subdivide en simple y obstructiva. Y es en el año 1975, bajo el padrinazgo de la American Thoracic Society y el American College of Chest Physicians, cuando surge por primera vez el acrónimo COPD, como una enfermedad de etiología incierta caracterizada por un persistente enlentecimiento al flujo aéreo durante la espiración forzada (2). Posteriormente también contó con una amplia difusión el término introducido por Thurlberck como obstrucción crónica al flujo aéreo (OCFA) (3). En el año 1987 la American Thoracic Society establece una definición morfológica y funcional de la EPOC como una alteración caracteri- zada por una disminución de los flujos aéreos espiratorios que no se modifican durante varios meses de observación y que está producida como consecuencia de alteraciones estructurales que afectan a las vías aéreas y al parénquima pulmonar, y que incluye la bronquitis crónica, el enfisema y la enfermedad de la vía aérea pequeña (4).

La enfermedad pulmonar obstructiva crónica (EPOC) es un proceso caracterizado por la presencia de una obstrucción crónica, progresiva y poco reversible al flujo aéreo, causado principalmente por una respuesta inflamatoria anómala frente al humo del tabaco (5).

$\mathrm{Si}$ hacemos algunas puntualizaciones a este respecto, diremos que dicho término surge para diferenciar este proceso de otras patologías que aunque cursan con reducción del flujo aéreo tienen una causa específica, como ocurre con la estenosis de la vía aérea superior, la fibrosis quística, las bronquiectasias, o la bronquiolitis obliterante. También lo diferencia de otros procesos como el asma bronquial, por su carácter rever-

Trabajo aceptado: 2 de noviembre de 2007 
sible (5). Finalmente haremos referencia a que no es un proceso que provoque exclusivamente el tabaco, sino que existen otros tóxicos inhalados que pueden producir EPOC pero que son muy poco frecuentes en nuestro medio (5).

La Neumopatía Obstructiva Crónica fue definida por la GOLD (Global Initiative for Chronic Obstructive Lung Disease) como un cuadro patológico caracterizado por una limitación del flujo de aire que no es totalmente reversible, habitualmente progresiva y relacionada con una respuesta inflamatoria anormal a partículas o gases nocivos. Incluye el enfisema, definido anatomopatológicamente como destrucción y ensanchamiento de los alvéolos pulmonares, y la bronquitis crónica, definida clínicamente por tos crónica productiva con un estrechamiento de los bronquíolos finos. Esta obstrucción al flujo aéreo deberá de ser duradera, pues la bronquitis crónica sin dicha limitación no se incluye (6).

Aunque el esqueleto fundamental de la definición se ha ido manteniendo con el paso de los años, los avances en el conocimiento tanto de la etiología como la patogenia, han hecho que el concepto también evolucionara. Si inicialmente se basaba en aspectos clínicos como tos y expectoración, posteriormente incorpora datos funcionales, como limitación al flujo aéreo, e histológicos, como enfisema o alteración de la vía aérea, para finalmente hacer referencia a la patogenia como una enfermedad de respuesta inflamatoria $(5,6)$.

No cabe duda de que el término EPOC es cuando menos polémico, y así lo manifestó Claude Lenfant en el prólogo del documento GOLD cuando hace referencia al escaso reconocimiento social de esta enfermedad (6).

Esta poco afortunada terminología, hace que se entienda mal fuera del mundo sanitario, lo que provoca que el paciente no sepa en muchas ocasiones precisar el origen o el curso de su enfermedad, y por otra parte el propio médico tenga dificultades para explicar el diagnóstico e incluso en otra ocasiones lo utilice como cajón de sastre donde meter a cualquier paciente con enfermedad respiratoria crónica. Es probable que una patología con unos índices de prevalencia y mortalidad tan importantes necesite una denominación más sencilla, cuya trasmisión al tejido social mediante las autoridades políticas y medios de comunicación, facilitara su conocimiento (7).

Otro de los factores que ha contribuido a las modificaciones que ha sufrido el término EPOC, y así convertirse en algo más concreto, ha sido el desarrollo tecnológico y muy directamente la tomografía axial computarizada de alta resolución (TACAR). Esta prueba ha permitido diferenciar de un modo claro las bronquiectasias de la EPOC, hecho que no resultaba tan sencillo cuando el diagnóstico se realizaba solo desde un punto de vista clínico (8).

En los pocos pacientes asmáticos con mala respuesta al tratamiento y escasa reversibilidad a en la espirometría (sobre todo si son fumadores) que pueden plantearnos dificultades de diagnóstico diferencial, también la TACAR ha contribuido a acotar el término EPOC, al definirse las manifestaciones radiológicas del remodelado bronquial con preservación del parénquima pulmonar como características del paciente asmático y así poder diferenciar ambas patologías (9).

Pero la TACAR no sólo ha contribuido a acotar el término, diferenciando patologías con límites en ocasiones superpuestos, para finalmente englobar a la bronquitis crónica, el enfisema pulmonar y la bronquiolitis obstructiva, También ha supuesto un pilar fundamental para diferenciar los tipos de enfisema, que desde el punto de vista etiológico y fisiopatológico parecen ser dos entidades distintas (10).

La etiopatogenia de la EPOC está íntimamente relacionada con el humo del tabaco y sin que haya muchas evidenciase rotundas también podrían influir factores ambientales y laborales (11). Es por ello que muchos autores hacen referencia a lo poco acertado del término en el sentido de que debería incluirse el daño y la causa en el diagnóstico, para de esta forma contribuir de forma más intensiva en la deshabituación tabáquica. Se han propuesto como alternativos los diagnósticos de "pulmón del fumador" o "tabacosis", existiendo datos de que al hacerlo así se produce una tasa de abandono del tabaco similar a la obtenida con programas intensivos de deshabituación (12).

Desde el punto de vista de la epidemiología se estima que la EPOC está infradiagnosticada. Para los más pesimistas los diagnosticados sólo suponen la punta de un iceberg que representaría el $25 \%$ de los casos, permaneciendo el otro $75 \%$ oculto (13). En el proyecto PADOC, en el que se realizó un cribaje de la EPOC en atención primaria entre población fumadora mayor de 35 años se objetivó alteración espirométrica en el $22 \%$ de las personas estudiadas (14). Al intentar analizar el problema, probablemente encontremos varios factores que han influido en ello. Por un lado la falta de síntomas importantes en las fases iniciales, unido a la falta de divulgación de la enfermedad en la población, a la que sin duda ha contribuido el no haber escogido un nombre más adecuado y preciso (15). Por otro lado, es el propio médico en muchas ocasiones el que no hace un diagnóstico certero, al no realizar las pruebas funcionales requeridas para objetivar la obstrucción persistente al flujo aéreo y cuantificar su grado. Recordemos que sólo el 49,1\% de los centros de atención primaria disponen de un espirómetro y de ellos sólo el $22,1 \%$ dispone de algún tipo de control de calidad periódico que certifique su fiabilidad (16). Pero independientemente de que se encuentren disponibles o no las pruebas complementarias necesarias, no existe una concienciación sistemática de búsqueda de obstrucción al flujo aéreo entre fumadores (17).

Es probable que en el futuro el nombre EPOC deba completarse con sus apellidos, que harán referencia a sus características fenotípicas, como el número de exacerbaciones anuales, grado de afectación músculo-esquelética (pérdida de masa muscular), percepción de los síntomas y capacidad funcional de ejercicio (calidad de vida) y sobre todo aquellos que se refieran a factores (además del tabaco) individuales (18). Confiemos en que en un futuro no muy lejano el conocimiento de la base genética de la enfermedad pueda identificar aquellos fumadores más susceptibles de padecer EPOC. Además contribuiría a no sólo considerarla como una enfermedad progresiva, en la que tan sólo disponemos de medidas paliativas, sino a soñar con tratamientos "curativos" con capacidad reparativa/regenerativa pulmonar (19). 


\section{Bibliografía}

1. Report of the conclusions of CIBA guest symposium: Terminology, definitions and classification of chronic obstructive pulmonary emphysema and related conditions. Thorax 1959; 14: 286-99.

2. American Thoracic Society. Definitions and classification of chronic bronchitis, asthma and pulmonary emphysema. Am Rev Respir Dis 1962; 85: 762-8.

3. Pulmonary terms and symbols. A report of the ACCP-ATS Joint Committee on Pulmonary Nomeclature. Chest 1975; 67: 583-93.

4. Thurlberck WM. Aspects of chronic airflow obstruction. Chest 1977; 72: 341-9

5. Barberá JA, Peces-Barba G, Agustí AGN, Izquierdo JL, Monsó E, Montemayor T, et al. Guía para el diagnóstico y tratamiento de la enfermedad pulmonar obstructiva crónica. Arch Bronconeumol 2001; 37: 297-316.

6. Pauwels RA, Buist AS, Calverley PMA, Jenkins CR, Hurd SS. Globa strategy for the diagnosis, management, and prevention of chronic obstructive pulmonary disease. NHLBI/WHO Global Initiative for Chronic Obstructive Lung Disease (GOLD). Workshop summary. Am J Respir Crit Care Med 2001; 163: 1256-76.

7. Rodríguez Roisín R, Álvarez -Sala JL, Sobradillo V. 2002: Un buen año capicúa para la EPOC. Arch Bronconeumol 2002; 38: 503-5.

8. O'Brien C, Guest PJ, Hill SL, Stockley RA. Physiological and radiological characterisation of patients diagnosed with chronic obstructive pulmonary disease in primary care. Thorax 2000; 55 : 635-42

9. Mochizuki T, Nakajima H, Kokubu F, Kushihashi T, Adachi M. Evaluation of emphysema in patients with reversible airway obstruction using high-resolution CT. Chest 1997; 112: 1522-6.
10. Bergin CJ, Muller NL, Miller RR, CT in the qualitative assessment of emphysema. J Thorac Imag 1986; 1: 94-103.

11. Hendrick DJ. Occupation and chronic obstructive pulmonary disease (COPD). Thorax 1996; 51: 947-55.

12. Brandt CJ, Ellegaard H, Joensen M, Kallan FV, Sorknaes AD, Tougaard L. Effect of diagnosis of "smoker's lung". RYLUNG Group. Lancet 1997; 349: 253.

13. Marco L, Martín JC, Corres M, Lique R, Zubillaga G. Enfermedad pulmonar obstructiva crónica en la población general. Estudio epidemiológico realizado en Guipúzcoa. Arch Bronconeumol 1999; 34: 23-7.

14. Miravitlles M, Fernández I, Guerrero T, Murio C. Desarrollo y resultados de un programa de cribado de la EPOC en Atención Primaria. Proyecto PADOC. Arch Bronconeumol 2000; 36: 500-5.

15. Snider GL. Nosology for our day: its implication to chronic obstructive pulmonary disease. Am J Respir Crit Care Med 2003; 167: 678-83.

16. De Miguel Díez J, Izquierdo Alonso JL, Molina París J, Rodríguez González-Moro JM, De Lucas Ramos P, Gaspar Alonso-Vega G. Fiabilidad del diagnóstico de la EPOC en atención primaria y neumología en España. Factores predictivos. Arch Bronconeumol 2003; 39: 203-8.

17. Croxton TL, Weinmann GG, Senior RM, Wise RA, Crapo JD, Buist AS. Clinical research in chronic obstructive pulmonary disease: Needs and opportunities. Am J Respir Crit Care Med 2003; 167: 1142-9.

18. Agustí AGN. Susceptibilidad a la EPOC. Arch Bronconeumol 2000; 36 (Supl. 3): 28-31

19. Massaro GD, Massaro D. Retinoic acid treatment abrogates elastaseinduced pulmonary emphysema in rats. Nat Med 1997; 3: 675-7. 